\title{
Characterization of the antibody response in birds following infection with wild-type and attenuated strains of Eimeria tenella and Eimeria necatrix
}

\author{
C.C. Constantinoiu ${ }^{\mathrm{a}, *}$, J.B. Molloy ${ }^{\mathrm{b}}$, W.K. Jorgensen ${ }^{\mathrm{b}}$, G.T. Coleman $^{\mathrm{a}}$ \\ a School of Veterinary Science, University of Queensland, St. Lucia, Brisbane, Queensland 4072, Australia \\ b Department of Primary Industries and Fisheries, 665 Fairfield Road, Yeerongpilly, Queensland 4105, Australia
}

\section{A R T I C L E I N F O}

\section{Article history:}

Received 17 April 2010

Received in revised form

14 September 2010

Accepted 24 September 2010

\section{Keywords:}

Eimeria

Strains

Chickens

Antibody response

Avidity

\begin{abstract}
A B S T R A C T
Live vaccines containing attenuated parasite strains are increasingly used to control chicken coccidiosis. In this paper antibody responses elicited by infections with wild-type and attenuated strains of Eimeria tenella and Eimeria necatrix were characterized by immunoblotting and ELISA with homologous and heterologous antisera. Few differences between antisera from birds infected with wild and attenuated strains of E. tenella were evident in immunoblots conducted with merozoite antigen preparations from both E. tenella strains, however the reactivity of sera raised in birds infected with the wild-type strain was noticeably more intense. In ELISAs conducted with merozoite antigen preparations, antisera from birds infected with the wild-type strains of E. tenella and E. necatrix consistently produced a significantly higher $(P<0.05)$ antibody response than antisera from birds infected with the attenuated strains. Likewise, avidity ELISAs conducted with the E. tenella strains demonstrated that antibodies in birds infected with the wild-type strain were of significantly higher avidity $(P<0.05)$ than antibodies in birds infected with the attenuated strain. The differences in the antibody responses are probably due to changes in the attenuated strain as a result of selection for precocious development and the less severe tissue damage and inflammation of the intestine resulting from infection with the attenuated strain.
\end{abstract}

Crown Copyright (c) 2010 Published by Elsevier B.V. All rights reserved.

\section{Introduction}

Coccidiosis is recognized as the parasitic disease with the greatest economic impact on poultry industries worldwide (Allen and Fetterer, 2002). The lifecycle of Eimeria comprises successive cycles of asexual development that are followed by sexual stages and release of oocysts in the faeces. In commercial production systems, coccidiosis is mainly controlled using chemical coccidiostats admin-

\footnotetext{
* Corresponding author. Present address: School of Veterinary and Biomedical Sciences, James Cook University, Townsville, Queensland 4811, Australia. Tel.: +61 74781 6635; fax: +61 747791526 .

E-mail address: constantin.constantinoiu@jcu.edu.au (C.C. Constantinoiu).
}

istered in feed (Shirley et al., 2005). However, increasing resistance to the available coccidiostats and consumer concern about residues in meat and eggs have resulted in increasing use of live vaccines containing attenuated parasite strains to control the disease (Chapman et al., 2002; Shirley et al., 2005).

Protective immunity against the parasites is thought to be predominantly cell-mediated, relying on T lymphocytes, natural killer cells and macrophages to control infection (Min et al., 2004; Dalloul and Lillehoj, 2005). However, the first subunit vaccine, $\operatorname{CoxAbic}^{\circledR}$, is based on transfer of protective antibodies from immunized hens to embryos (Belli et al., 2004), suggesting that antibodies play a role in immunity under certain conditions. Furthermore, antibodies can be useful as diagnostic tools to determine the exposure of individual birds or flocks to Eimeria spp. or for monitoring 
vaccination programmes (Onaga et al., 1986; Smith et al., 1993; Constantinoiu et al., 2007).

The antibody responses of chickens to Eimeria infections have been characterized with regard to parasite lifecycle stages, immunodominant antigens and dynamics (Xie et al., 1992; Smith et al., 1993; Tomley, 1994; Talebi and Mulcahy, 1995; Tajima et al., 2003; Onaga et al., 2005; Constantinoiu et al., 2007). However, most studies of the antibody responses reported to date have been conducted with wild-type strains that have not been subject to any selection. Attenuated strains, on the other hand, are often subjected to rigorous selection for precocious development that can result in abbreviation of some lifecycle stages (Shirley et al., 2005). Here we report on the characterization of the antibody response elicited by infections with both wild-type and attenuated strains of Eimeria tenella and Eimeria necatrix.

\section{Materials and methods}

\subsection{Birds and experimental groups}

Rhode Island Red/Rhode Island White cross chickens were purchased from a commercial supplier as day-old chickens and housed in positive pressure isolators with HEPA filtered air until 4-6 weeks of age. While in the isolators the birds were maintained on feed containing a commercial coccidiostat (Cycostat 66, Roche, Frenchs Forest) at $0.5 \mathrm{~g} / \mathrm{kg}$, and faeces were tested weekly by the sugar flotation test to confirm freedom from Eimeria spp. (Jorgensen et al., 2006). For the experimental work the birds were transferred to wire cages in closed climate controlled rooms with restricted access. All experiments involving live chickens were performed with the approval of the Animal Research Institute Animal Ethics Review Committee (Approved Application Number: ARI29/06/2005).

\subsection{Parasites}

Two strains of E. tenella (Ingten and Redlands), and three strains of E. necatrix (Gronec, Mednec and Mednec 3+8) (Jorgensen and Anderson, 1999; Jorgensen et al., 2006) were used in these experiments. Oocysts were sporulated, separated from the faeces and stored as described previously (Jorgensen et al., 1997). The Relands strain of E. tenella and the Mednec $3+8$ strain of E. necatrix had been previously attenuated by passage in susceptible birds and selection for precocious development (Jorgensen and Anderson, 1999; Jorgensen et al., 2006). All the other strains were wild-type strains.

\subsection{Sera}

For production of immune sera against E. tenella, 11 birds per group were inoculated by crop gavage with 1500 sporulated oocysts of the Ingten strain or 3000 oocysts of the Redlands strain in a $1 \mathrm{ml}$ dose, three times at two-week intervals. For $E$. necatrix, eight birds were inoculated with the Gronec strain, four with the Mednec strain and six with the Mednec $3+8$ strain. The birds from the E. necatrix group were inoculated with 3000 sporulated oocysts, three times at two-week intervals (Gronec and Mednec 3+8 strains). The birds inoculated with the Mednec strain were inoculated as those with Gronec and Mednec 3+8 except that the first inoculation was carried out with 1200 sporulated oocysts. Different inocula were used to compensate for the lower reproductive potential of the attenuated strains and the extreme virulence of some of the wild strains. Negative control groups of two birds were mock inoculated three times at two-week intervals with $1 \mathrm{ml}$ of distilled water. Throughout the experiments trays were placed in the bottom of the cages to facilitate oocyst recirculation and to simulate conditions that occur on commercial farms where birds are reared on litter.

Three weeks after the last inoculation all birds were anaesthetised with isoflurane (IsoFlo, Abbot Australia), heart bled and euthanized by cervical dislocation before recovery. Sera were aliquoted and stored at $-20^{\circ} \mathrm{C}$ until use.

\subsection{Antigen preparation, protein separation and Western blotting}

E. tenella Ingten and Redlands strain merozoites from the last generation were obtained as described by Shirley (1995). Preparation of antigens from purified merozoites, protein separation by SDS-PAGE and protein transfer on PVDF membranes were carried out as previously described (Constantinoiu et al., 2007, 2008). Further, the membranes were blocked with $2 \%(\mathrm{w} / \mathrm{v})$ skim milk powder (SMP) in $0.05 \%(\mathrm{v} / \mathrm{v})$ Tween 20 in Tris-saline $(0.01 \mathrm{M}$ Tris, $0.154 \mathrm{M}$ $\mathrm{NaCl}, \mathrm{pH}$ 7.4) (TTS) for one hour at room temperature (RT), washed once with TTS then incubated with chicken sera diluted $1: 25(\mathrm{v} / \mathrm{v})$ in $2 \%(\mathrm{w} / \mathrm{v})$ SMP in TTS for $1 \mathrm{~h}$ at RT. Membranes were then washed three times in TTS and incubated for $1 \mathrm{~h}$ at RT with goat anti-chicken IgG HRP conjugate (Bethyl Laboratories Inc., Montgomery, TX, USA) diluted $1: 400(\mathrm{v} / \mathrm{v})$ in $2 \%(\mathrm{w} / \mathrm{v})$ SMP in TTS. After three final washes with TTS and one with Tris-saline $(0.01 \mathrm{M}$ Tris, $0.154 \mathrm{M}$ $\mathrm{NaCl}, \mathrm{pH}$ 7.4) the colour reaction was developed with 4chloro-1-naphtol (Bio-Rad, Hercules, CA, USA) for $2 \mathrm{~min}$.

\subsection{ELISA}

Sera from birds infected with the E. tenella strains were tested in an ELISA using merozoite antigens from both the Redlands and Ingten strains as previously described (Constantinoiu et al., 2007). ELISAs with sera from birds infected with the E. necatrix strains were conducted using merozoite antigens from the Redlands strain of E. tenella. Briefly, test wells of microtitre plates (MaxiSorp, Nunc, Roskilde, Denmark) were coated overnight, at $4{ }^{\circ} \mathrm{C}$, with $1 \mu \mathrm{g}$ of merozoite antigen in $100 \mu \mathrm{l}$ of $0.05 \mathrm{M}$ carbonate buffer ( $\mathrm{pH}$ 9.6). Control wells were incubated overnight at $4^{\circ} \mathrm{C}$, with $100 \mu \mathrm{l}$ of $0.05 \mathrm{M}$ carbonate buffer ( $\mathrm{pH} 9.6$ ) only. Vacant binding sites were then blocked by incubation with $200 \mu \mathrm{l}$ of $2 \%(\mathrm{w} / \mathrm{v}) \mathrm{SMP}$ in phosphate-buffered saline (136.8 mM NaCl, $1.47 \mathrm{mM} \mathrm{KH}_{2} \mathrm{PO}_{4}, 8 \mathrm{mM} \mathrm{Na}_{2} \mathrm{HPO}_{4}$ and $2.68 \mathrm{mM} \mathrm{KCl}, \mathrm{pH} 7.4$, PBS) containing $0.05 \%$ Tween 20 (PBST) for $1 \mathrm{~h}$ at RT. After three washes with PBST the wells were incubated for $1 \mathrm{~h}$ at RT on a shaker with 


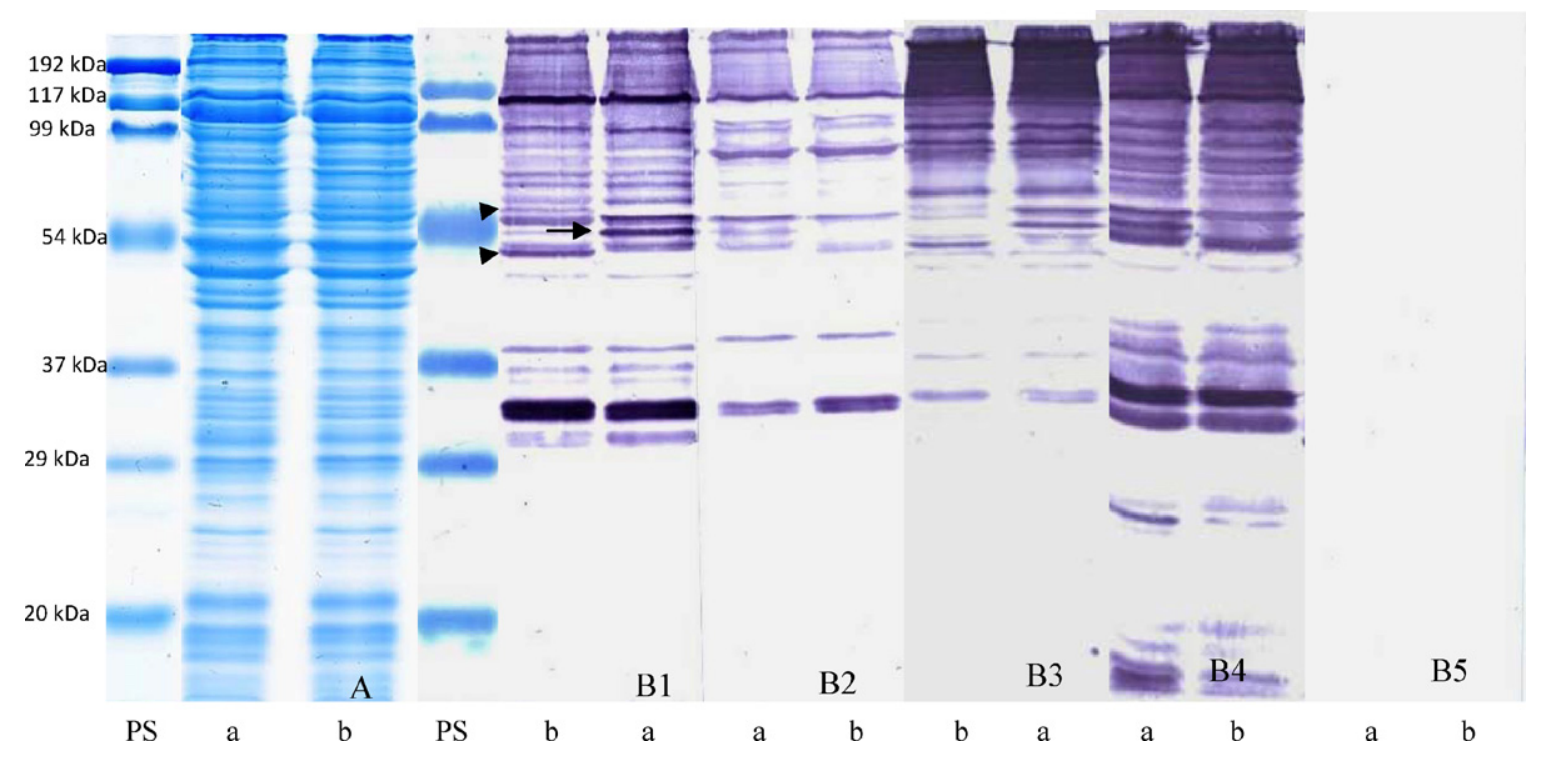

Fig. 1. Merozoite proteins of Ingten (a) and Redlands (b) E. tenella strains resolved by SDS-PAGE on a $12 \%$ gel and stained by Coomassie blue brilliant (A) or probed in immunoblots with sera from two birds infected with the Ingten strain parasites (B1 and B4), two birds infected with the Redlands strain parasites (B2 and B3) and a single uninfected birds (B5). The arrows show the 50.5, 55 and 60.5 kDa band differentially represented in the two strains. PS stands for protein standards.

sera diluted 1:100 (v/v) in $2 \%(\mathrm{w} / \mathrm{v})$ SMP in PBST. After the plates were washed five times with PBST the wells were filled with $100 \mu \mathrm{l}$ of goat anti-chicken IgG HRP conjugate (Bethyl Laboratories Inc., Montgomery, TX, USA) diluted in $1: 50,000(\mathrm{v} / \mathrm{v})$ in $2 \%(\mathrm{w} / \mathrm{v})$ SMP in PBST. After $1 \mathrm{~h}$ incubation at RT on a shaker the plates were washed five times with PBST and the enzyme reaction developed with 3,3',5,5'-tetramethylbenzidine (TMB) (KPL, Gaithersburg, MD, USA) for $10 \mathrm{~min}$ in the dark. The reaction was stopped by the addition of $100 \mu \mathrm{L}$ of $2 \mathrm{M} \mathrm{H}_{3} \mathrm{PO}_{4}$ per well and the absorbance read at $450 \mathrm{~nm}$ with an ELISA Microplate Reader (Model 680, Bio-Rad Laboratories, Hercules, CA, USA). All sera were analysed in duplicate. For each serum, the average absorbance of the uncoated wells was then subtracted from the average absorbance of the antigencoated wells. Serum from a bird infected with the Redlands strain of $E$. tenella produced one of the highest absorbances, and was chosen as the positive control in all ELISAs. Absorbances of all other sera were calculated as percentage of that positive control and expressed as percent positivity (PP).

\subsection{Antibody avidity in ELISA}

The avidity of antibodies for the parasite antigens was determined by ELISA as described by Prince and Wilson (2001). Briefly, after the serum incubation stage, weakly bound specific antibodies were removed by two 5 min incubations with $6 \mathrm{M}$ urea (Sigma, St. Louis, MO, USA) diluted in phosphate-buffered saline $(136.8 \mathrm{mM} \mathrm{NaCl}, 1.47 \mathrm{mM}$ $\mathrm{KH}_{2} \mathrm{PO}_{4}, 8 \mathrm{mM} \mathrm{Na}_{2} \mathrm{HPO}_{4}$ and $2.68 \mathrm{mM} \mathrm{KCl}, \mathrm{pH}$ 7.4) containing $0.05 \%$ Tween 20 (PBST). Control wells were similarly incubated with (PBST) only. Percentage avidity (PA) was calculated as described by Maley et al., 2001 (percentage avidity $=\mathrm{OD}$ (urea treated)/OD (untreated) $\times 100$ ).

\subsection{Statistical analyses}

The untransformed PP data were subjected to two-way analysis of variance in GenStat (2005), with 'parasite type' as the factor. The interaction term was included, and individual birds were taken as the experimental units.

\section{Results}

\subsection{Immunoblots of merozoite antigens from the Ingten and Redlands strains of E. tenella}

Antibody reactions in sera from chickens inoculated with the Ingten or Redlands strains were compared by SDS-PAGE and immunoblotting with purified merozoite antigen preparations from homologous or heterologous strains. Two sera from birds inoculated with either Ingten or Redlands strains were used to probe merozoite proteins in immunoblots. In Coomassie brilliant blue stained gels, the protein profiles of the two merozoite strains were complex but virtually identical (Fig. 1). In immunoblots, sera collected from birds infected with the Redlands strain of $E$. tenella recognized a lower number of antigens of both parasite strains than sera obtained from birds infected with the Ingten strain. In addition, the intensity of the reaction with common bands was less intense with the Redlands antisera (Fig. 1). A dominant protein of $34 \mathrm{kDa}$ was recognized by all sera in merozoites of both parasites strains. However, two antigens of $50.5 \mathrm{kDa}$ and $60.5 \mathrm{kDa}$ seemed to be present only in the Redlands strain merozoites. On the other hand, a $55 \mathrm{kDa}$ antigen present in the Ingten merozoites and was absent or very weak in the Redlands strain merozoites.

\subsection{Quantification of the antibody response by ELISA}

Antibody reactions in sera from all chickens inoculated with the two strains of E. tenella were compared by 


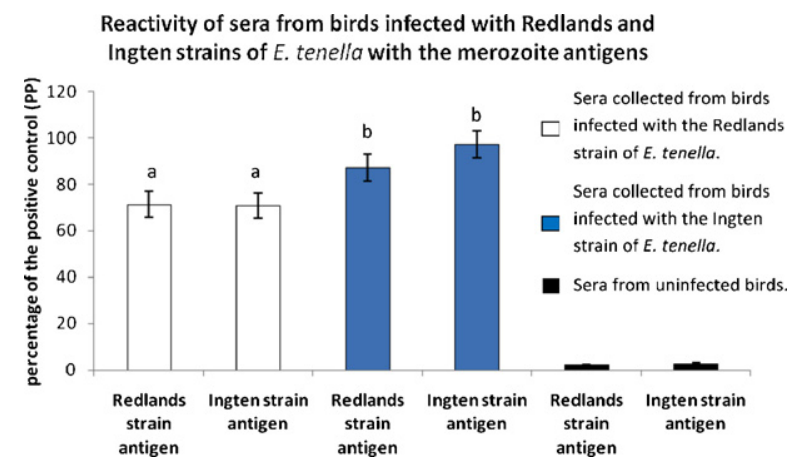

Fig. 2. Antibody response in birds infected with the Redlands and Ingten strains of E. tenella in ELISA. Merozoite antigens from the two strains of parasites were reacted with homologous and heterologous antisera. Average of percentage positivity (PP) and standard error bars for 11 sera from birds infected with the Redlands or Ingten strains and 4 sera from uninfected birds are presented. Different letters (a or b) indicate significant differences at $P<0.05$.

ELISA using merozoite antigen preparations of both parasite strains. For both antigen preparations the antibody response elicited by infection with the Ingten strain was significantly higher $(P<0.05)$ than the response to infection with the Redlands strain (Fig. 2). In birds infected with the Ingten or Redlands strain parasites, the antibody levels against the two merozoites antigen preparations were not significantly different $(P<0.05)$ (Fig. 2). The antibody levels elicited by infection with the three strains of $E$. necatrix were quantified against the merozoite antigen preparation of the Redlands strain of E. tenella (Fig. 3). The antibody response in birds infected with the Gronec and Mednec strains was significantly higher $(P<0.05)$ than the response in birds infected with the Mednec $3+8$ strain (Fig. 3).

\subsection{Antibody avidity}

The avidity of antibodies against both antigen preparations was determined in sera from birds infected with the two strains of E. tenella. Absorbances of sera were

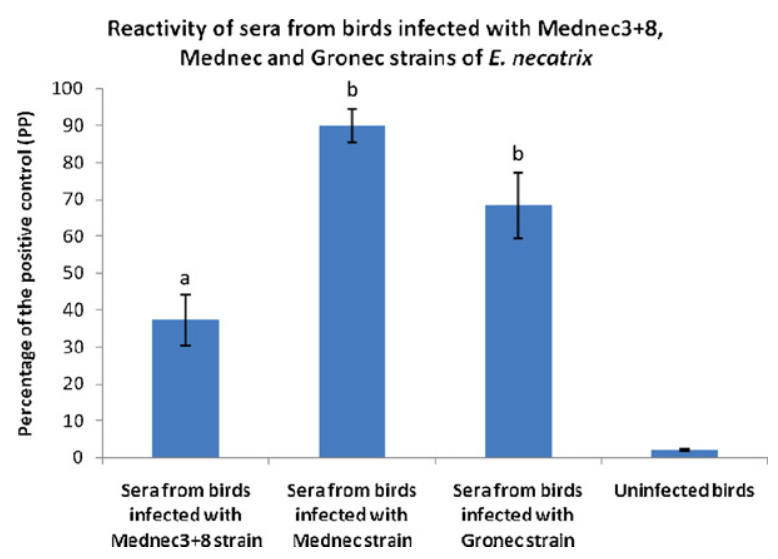

Fig. 3. Antibody response in birds infected with the Mednec 3+8, Mednec and Gronec strains of E. necatrix against the merozoite antigen from the Redlands strain of E. tenella in ELISA. Average of percentage positivity (PP) and standard error bars are presented. Different letters (a or b) indicate significant differences at $P<0.05$.

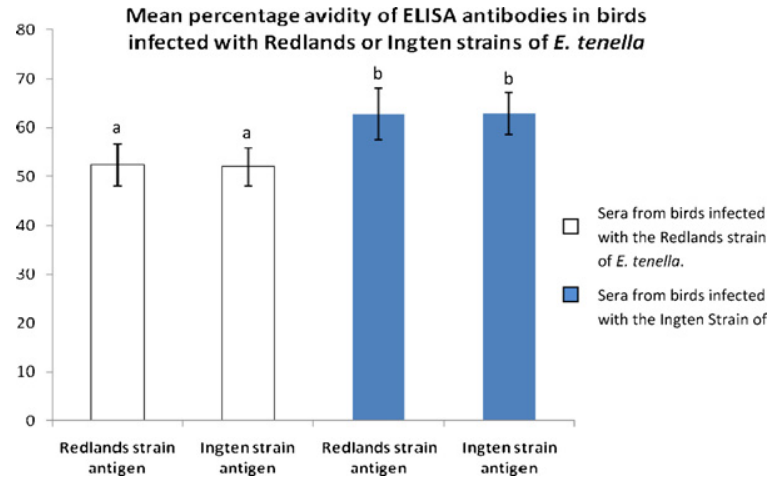

Fig. 4. Mean percentage avidity of ELISA antibodies in birds infected with the Redlands or Ingten strains of E. tenella. Different letters ( $a$ or b) indicate significant differences at $P<0.05$.

reduced after low avidity antibodies were removed by treatment with urea (Fig. 4). For each group of sera (from birds infected with either the Ingten or with the Redlands strain parasites) the reduction in the absorbances of the urea treated sera was similar for the two merozoite antigen preparations. Thus, for the Redlands strain merozoite antigen the reduction in the mean OD of sera from birds inoculated with the Redlands strain parasites was from 1.02 to 0.55 while for the sera from birds inoculated with the Ingten strain of parasites the reduction in the mean OD was from 1.61 to 1.04. Similarly, for the Ingten strain merozoites antigen the reduction in the mean OD of sera from birds inoculated with the Redlands strain of parasites was from 1.19 to 0.64 while for the sera from birds inoculated with Ingten strain of parasites the reduction in the mean OD of sera was from 1.87 to 1.21 . The avidity of antibodies in sera of birds inoculated with the wild-type Ingten strain parasites was significantly higher $(P<0.05)$ than that of antibodies from birds inoculated with the attenuated strain parasites (Fig. 4).

\section{Discussion}

No differences between the antigen profiles of the Ingten and Redlands strain merozoites were evident on the stained gels and the vast majority of antigens recognized in immunoblots with homolgous and heterologous antisera were common to both strains. However, the intensity of the reaction was stronger with sera collected from birds infected with the wild-type Ingten strain parasites than with the attenuated Redlands strain, suggesting a stronger antibody response against the wild-type strain. ELISA data from birds infected with wild-type and attenuated strains of E. tenella and E. necatrix appeared to confirm this interpretation. Furthermore, the avidity of antibodies from birds infected with Ingten strain parasites was significantly higher than that of antibodies from birds infected with the Redlands parasites.

Our data show that there are differences in the intensity of the antibody response in birds infected with two different strains of $E$. tenella. The most likely explanation for the difference is that Ingten is a virulent wild-type strain whereas the Redlands strain is an attenuated strain 
obtained by selection for precocious development. Such selection has been shown to result in a decreased number of schizogonic generations that mature faster and produce smaller schizonts, with reduced numbers of merozoites and consequent decreased reproductive potential (Shirley and Long, 1990) which could result in a less intense antibody response in infected birds. Furthermore, the virulent strains might induce more severe tissue damage and inflammation of the intestine with consecutive recruitment of immune cells at the site of infection.

Even though the antibody responses elicited by infections with the attenuated strains of $E$. tenella and $E$. necatrix were lower than that elicited by the virulent strains the attenuated strains have been shown to be protective against challenges with homologous and heterologous strains (Jorgensen et al., 2006) which supports the general belief that under most circumstances the role of antibodies in protection is minimal (Rose and Wakelin, 1990). On the other hand these differences in the antibody response should be considered when developing and evaluating serological tests for the diagnosis of Eimeria infections. The implications of differences in the avidity of antibodies in the sera of birds inoculated with different strains of E. tenella are difficult to interpret as, to our knowledge, there are no previous reports of high or low avidity antibodies in Eimeria infections or the dynamics of the evolution of high avidity antibodies in chronic infections. In any event, the finding that attenuated strains tend to produce lower avidity antibodies could also have implications for the development and evaluation of serological tests, particularly when they are to be used to test birds with recent infections with vaccine strains.

\section{Acknowledgments}

Special thanks to David Mayer for statistical analysis of the data and Anthea Bruyeres, Sandy Jarrett, Jan-Maree Hewitson, Ryan O'Neil, Andrew Kelly and Ashley Ostrofsky for able technical assistance.

\section{References}

Allen, P.C., Fetterer, R.H., 2002. Recent advances in biology and immunobiology of Eimeria species and in diagnosis and control of infection with these coccidian parasites of poultry. Clin. Microbiol. Rev. 15, 58-65.

Belli, S.I., Mai, K., Skene, C.D., Gleeson, M.T., Witcombe, D.M., Katrib, M., Finger, A., Wallach, M.G., Smith, N.C., 2004. Characterisation of the antigenic and immunogenic properties of bacterially expressed, sexual stage antigens of the coccidian parasite, Eimeria maxima. Vaccine 22, 4316-4325.
Chapman, H.D., Cherry, T.E., Danforth, H.D., Richards, G., Shirley, M.W., Williams, R.B., 2002. Sustainable coccidiosis control in poultry production: the role of live vaccines. Int. J. Parasitol. 32, 617-629.

Constantinoiu, C.C., Molloy, J.B., Jorgensen, W.K., Coleman, G.T., 2007. Development and validation of an ELISA for detecting antibodies to Eimeria tenella in chickens. Vet. Parasitol. 150, 306-313.

Constantinoiu, C.C., Molloy, J.B., Jorgensen, W.K., Coleman, G.T., 2008. Antibody response against endogenous stages of an attenuated strain of Eimeria tenella. Vet. Parasitol. 154, 193-204.

Dalloul, R.A., Lillehoj, H.S., 2005. Recent advances in immunomodulation and vaccination strategies against coccidiosis. Avian Dis. 49, 1-8.

Jorgensen, W.K., Stewart, N.P., Jeston, P.J., Molloy, J.B., Blight, G.W., Dalgliesh, R.J., 1997. Isolation and pathogenicity of Australian strains of Eimeria praecox and Eimeria mitis. Aust. Vet. J. 75, 592-595.

Jorgensen, W.K., Anderson, G.R., 1999. Attenuation and characterization of Eimeria spp. A report for the Rural Industries Research and Development Corporation. RIRDC Project DAQ-215J. RIRDC Publication No. 98, ACT, Australia.

Jorgensen, W.K., Anderson, G.R., Jeston, P.J., Blight, G.W., Molloy, J.B., 2006. Selection and characterisation of two attenuated vaccine lines of Eimeria tenella in Australia. Aust. Vet. J. 84, 89-94.

Maley, S.W., Buxton, D., Thomson, K.M., Schriefer, C.E., Innes, E.A., 2001. Serological analysis of calves experimentally infected with Neospora caninum: a 1-year study. Vet. Parasitol. 96, 1-9.

Min, W., Dalloul, R.A., Lillehoj, H.S., 2004. Application of biotechnological tools for coccidia vaccine development. J. Vet. Sci. 5, 279-288.

Onaga, H., Saeki, H., Hoshi, S., Ueda, S., 1986. An enzyme-linked immunosorbent assay for serodiagnosis of coccidiosis in chickens: use of a single serum dilution. Avian Dis. 30, 658-661.

Onaga, H., Kawahara, F., Umeda, K., Nagai, S., 2005. Field basis evaluation of Eimeria necatrix-specific enzyme-linked immunosorbent assay (ELISA) for its utility in detecting antibodies elicited by vaccination in chickens. J. Vet. Med. Sci. 67, 947-949.

Prince, H.E., Wilson, M., 2001. Simplified assay for measuring Toxoplasma gondii immunoglobulin G avidity. Clin. Diagn. Lab. Immunol. 8, 904-908.

Rose, M.E., Wakelin, D., 1990. Immunity to Coccidiosis. In: Long, P.L. (Ed.), Coccidiosis of Man and Domestic Animals. CRC Press, Boca Raton, Florida, pp. 281-307.

Shirley, M.W., 1995. Eimeria species and strains of chickens. In: Eckert, J., Braun, R., Shirley, M.W., Coudert, P. (Eds.), Guidelines on Techniques in Coccidiosis Research. European Commission, pp. 1-25.

Shirley, M.W., Long, P.L., 1990. Control of Coccidiosis in chickens: immunization with live vaccines. In: Long, P.L. (Ed.), Coccidiosis of Man and Domestic Animals. CRC Press, Boca Raton, Florida, pp. 321-341.

Shirley, M.W., Smith, A.L., Tomley, F.M., 2005. The biology of avian Eimeria with an emphasis on their control by vaccination. Adv. Parasitol. 60, 285-330.

Smith, N.C., Bucklar, H., Muggli, E., Hoop, R.K., Gottstein, B., Eckert, J., 1993. Use of IgG- and IgM-specific ELISAs for the assessment of exposure status of chickens to Eimeria species. Vet. Parasitol. 51, 13-25.

Tajima, O., Onaga, H., Nakamura, T., 2003. An enzyme-linked immunosorbent assay with the recombinant merozoite protein as antigen for detection of antibodies to Eimeria necatrix. Avian Dis. 47, 309318.

Talebi, A., Mulcahy, G., 1995. Cross-reactivity among antisera raised against five avian Eimeria species in the natural host and in rabbits. Avian Pathol. 24, 533-544.

Tomley, F., 1994. Antigenic diversity of the asexual developmental stages of Eimeria tenella. Parasite Immunol. 16, 407-413.

Xie, M., Gilbert, J.M., McDougald, L.R., 1992. Electrophoretic and immunologic characterization of proteins of merozoites of Eimeria acervulina, E. maxima, E. necatrix, and E. tenella. J. Parasitol. 78, 82-86. 